\title{
Diagnosing Genitourinary Tuberculosis: A Case Report
}

\author{
Chitsimran Mann', Gina Maki, DO' ${ }^{2}$, Marcus Zervos, MD ${ }^{2,3}$ and Nidhi Ravishankar ${ }^{*}$ \\ ${ }^{1}$ Department of Internal Medicine, Windsor University School of Medicine, USA \\ ${ }^{2}$ Department of Infectious Diseases, Henry Ford Health System, Detroit, Michigan, USA \\ ${ }^{3}$ Wayne State University School of Medicine, Detroit, MI, USA
}

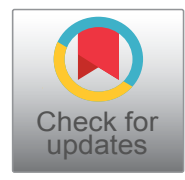

*Corresponding author: Nidhi Ravishankar, Department of Internal Medicine, Windsor University School of Medicine, 6212 Monee Manhattan Road, Monee, IL 60449, USA, Tel: 3125082025

\begin{abstract}
Genitourinary Tuberculosis (GUTB) is a common manifestation of tuberculosis and usually results from the reactivation of old, dormant tuberculous diseases by pathogens of the Mycobacterium tuberculosis complex. Most often, GUTB has a varied presentation ranging from irritative voiding symptoms to renal failure. Due to this varied presentation, diagnosis of GUTB is often very difficult. We present a case of a reproductive-age female with asymptomatic infertility. Many diagnostic measures failed to identify the root cause of her infertility, however the positive PPD test was the first indicator of a possible reactivation of tuberculosis and histopathological examination of the tissue confirmed the diagnosis. We describe the definitive measure used to diagnose GUTB and the improvement of her infertility after anti-tubercular medications.
\end{abstract}

\section{Introduction}

Tuberculosis is one of the largest causes of mortality worldwide. Approximately one quarter of the world's population has latent tuberculosis (TB) [1]. While pulmonary TB is the most common presentation (69.6\%), about $1 / 5(20.3 \%)$ cases are of extrapulmonary tuberculosis (EPTB). Common EPTB presentations included lymphatic (35.8\%), pleural (16.1\%), bone and joint (9.7\%), genitourinary (4.6\%), meningeal (4.2\%), and peritoneal (6.1\%) [2]. Genitourinary TB (GUTB) is a frequent manifestation of tuberculosis that is often overlooked.

GUTB is an infectious inflammation of urogenital organs, isolated or in combination (kidney, urinary tract, and/or male or female genitals), caused by Mycobacterium tuberculosis or M. bovis [3] with or without pulmonary involvement. In females, involvement of the uterus and fallopian tubes cause strictures and scaring leading to primary infertility, secondary infertility or ectopic pregnancy [4].

GUTB presents a diagnostic problem. As per the World Health Organization (WHO), EPTB can be diagnosed based on one culture-positive specimen, or positive histology or strong [2] clinical evidence consistent with active EPTB [5]. It can take up to six weeks for a culture to become positive. Using PCR, M. tuberculosis DNA was detected in up to $80.9 \%$ of suspected cases with a sensitivity of $87-100 \%$ and a specificity of $92.2-$ $98 \%$ [6-8]. We report a case of a female presenting with infertility with no diagnostic indication of tuberculosis.

\section{Case Report}

A 24-year-old nulliparous woman, immigrant of India, with no significant past medical history presented with a chief complaint of infertility. Patient was married for 5 years and unable to conceive despite having regular unprotected intercourse. Patient had regular (30 days) menstrual cycles, with no other related complaints.

Home medications included aspirin for occasional headaches. She denied having any known drug allergies. She was a lifetime nonsmoker, did not drink alcohol, or use illicit drugs. Patient had received all immunizations in the past but did not receive the BCG vaccine.

Physical examination revealed a healthy woman with a temperature of $36.6^{\circ} \mathrm{C}\left(97.8^{\circ} \mathrm{F}\right)$, blood pressure of $123 / 81 \mathrm{mmHg}$, pulse rate of 89 beats/min, and respiratory rate of $17 \mathrm{breaths} / \mathrm{min}$. The rest of the examination was notable for a minimally distended non-tender 
abdomen, no rebound sign or voluntary guarding in all four quadrants. Notable laboratory values were as follows: serum sodium level, $139 \mathrm{mEq} / \mathrm{mL}$ (normal = 135$145 \mathrm{mEq} / \mathrm{L}$; potassium level, $3.8 \mathrm{mmol} / \mathrm{mL}$ (normal = 3.5-5 mEq/L); hemoglobin level, $9.6 \mathrm{~g} / \mathrm{dL}$ (normal = 12$15.5 \mathrm{~g} / \mathrm{dL}$ ); hematocrit, $33.7 \%$ (normal $=37-48 \%$ ); and WBC count, 6200 cells $/ \mathrm{mm}^{3}$ (normal $=4500-11000$ cells ) $\mathrm{mm}^{3}$ ) , with $56 \%$ neutrophils (normal $=45-70 \%$ ) and $2 \%$ band cells (normal $=<10 \%$ ), ESR of $55 \mathrm{~mm} / \mathrm{hr}$ (normal = $0-20 \mathrm{~mm} / \mathrm{hr}$ ). The total serum protein level was $9 \mathrm{~g} / \mathrm{dL}$ (normal $=6-8 \mathrm{~g} / \mathrm{dL}$ ) and the albumin level was $4.1 \mathrm{~g} / \mathrm{dL}$ (normal $=3.5-5.5 \mathrm{~g} / \mathrm{dL}$ ). Thorough infertility testing was performed including hormonal panels, ultrasound, hysterosalpingogram, hysteroscopy, endometrial biopsies and cultures, and semen analysis. Testing revealed no abnormalities. Diagnostic testing for tuberculosis was conducted due to the high prevalence of extra-pulmonary tuberculosis in India. Although the PPD was $16 \mathrm{~mm}$, subsequent histopathological and radiological tests for confirmation with urine AFB stain, urine AFB culture and urine tuberculosis polymerase chain reaction (UT-PCR) were negative. The subsequent chest XR was clear.

The commercially available Quantiferon-TB gold intube kit (Cellestis) was used to facilitate the Interferon- $y$ Release Assay (IGRA). $1 \mathrm{~mL}$ of whole blood was added to each of the three QFT-TB tubes: The TB antigen tube (ESAT-6, CEP-10, and TB 7.7), the mitogen-positive control tube (phytohemagglutinin), and the negative control tube. Plasma was measured by the IFN-gamma. An IFN-gamma concentration $\geq 0.35 \mathrm{IU} / \mathrm{mL}$ (TB antigen minus negative control) is considered a positive test result [9]. Patient had a concentration of $0.1 \mathrm{IU} / \mathrm{mL}$.

Subsequent radiological examination using MRI imaging of pelvis and lower abdomen was performed, and $\mathrm{T} 1$ and $\mathrm{T} 2$ weighted serial sections obtained in the sagittal, axial and coronal planes using a dedicated torso-array surface coil and respiratory compensation was examined. On the MRI, a complex thick-walled cyst with blood contents was seen separate from both ovaries in the pelvis as well as mild ascites.

Laproscopy was performed under general anesthesia using $0^{\circ}, 10 \mathrm{~mm}$ laparoscope. Along with scarred Omentum, round caseating masses were observed in the pelvis to which the fimbrial end of both the fallopian tubes were adherent. Histologic examination of the mass showed caseating granulomatous inflammation with Langhans giant cells at 10x suggestive of calcified tuberculosis. The patient was treated with a six-month course of RIPE (rifampin, isoniazid, pyrazinamide, ethambutol). At present, patient is currently 3 months pregnant.

\section{Discussion}

Our patient represented a case of tuberculosis presenting with infertility and no other symptoms. Generally, tubercle bacilli are inhaled through droplets that reach the alveoli of lungs where they are ingested by alveolar macrophages. Usually, most of these bacilli are destroyed, but a small number may remain that later multiply intracellularly. These bacilli are released when the macrophage dies and spreads via the lymphatic channels through the bloodstream to distant tissues and organs. Previous cases of GUTB included symptoms of dysmenorrhea, dyspareunia, amenorrhea (primary and secondary), menorrhagia and postmenopausal bleeding [10]. Genitourinary TB can mimic malignancy and even pregnancy when coupled with amenorrhea, hence, definite diagnosis of the primary cause of amenorrhea is extremely necessary.

For a definite diagnosis of GUTB, microbiological and/or histopathological confirmation is required. Histopathology examination (HPE) is routinely used for diagnosis of GUTB as it is simple, quick and economical [11]. In our case, the HPE of the endometrial biopsy was negative but HPE of the caseating mass proved to be diagnostic.

Gene amplification using polymerase chain reaction (PCR) to detect $M$. tuberculosis on ascitic fluid and peritoneal biopsies are another rapid and non-invasive test used previously [12]. However, low sensitivity has been reported in smear-negative patients (48\%), as seen with our patient. Similary, IFN- $\gamma$ release assay might feasibly be used as a supplementary or screening tool for the diagnosis of GUTB but due to its undefined success rates, further studies for statistical evaluation of its sensitivity, specificity, and efficacy are needed [13]. This means that in majority cases, like this case, diagnostic laparoscopy with abdominal imaging and direct visualization of the peritoneum and biopsy may be crucial for diagnosis.

Laparoscopy is easily accessible, relatively safe with a reported complication rate of $<3 \%$ and a high diagnostic sensitivity (93\%) and specificity (98\%) [12]. In our case, laparoscopy allowed for direct visualization and biopsy of the para-uterine cystic formation described on abdominal MRI. Upon histopathology of the fimbrael biopsy attained, tuberculosis was confirmed and anti-tubercular treatment was started.

A small number of TB bacilli can cause a great amount of damage due to the vulnerability of the area involved. Initially, small nodules of tubercular material deposit on the tubular surface. Later, the infection invades the tubes, affecting the internal surface. Pus collects within the tubes, blocking them either completely or partially, resulting in a condition known as hydro-salpingitis [14]. Sterility and subfertility/infertility is the most common complication owing to involvement of ovaries (eg. masses or cysts), fallopian tubes (congestion \& scarring) and endometrium (eg Asherman syndrome) even after treatment. Complications such as peritonitis, pneumoperitoneum, and bladder injury can be caused during diagnostic procedures. Screening for GUTB in 
patients of reproductive age presenting with infertility, pelvic pain, and menstrual dysfunction is important when other causes have been ruled out. Unfortunately, most patients presenting with advanced disease exhibit severe fibrosis and adhesions leading to poor fertility outcomes. Early diagnosis and proper treatment is important to avoid complications, mortality and restore fertility.

\section{References}

1. Houben RM, Dodd PJ (2016) The global burden of latent tuberculosis infection: A Re-estimation using mathematical modelling. PLoS Med 13: e1002152.

2. (2016) Reported Tuberculosis in the United States.

3. Kulchavenya E (2016) Current therapy and surgery for urogenital tuberculosis. Springer Berlin Heidelberg, New York, NY.

4. Sharma N, Sharma V, Singh PR, Sailwal S, Kushwaha $R S$, et al. (2013) Diagnostic value of PCR in genitourinary tuberculosis. Indian J Clin Biochem 28: 305-308.

5. World Health Organization (2013) Global tuberculosis report 2013.

6. Tostmann A, Kik SV, Kalisvaart NA, Sebek MM, Verver $S$, et al. (2008) Tuberculosis transmission by patients with smear-negative pulmonary tuberculosis in a large cohort in the Netherlands. Clin Infect Dis 47: 1135-1142.

7. Moussa OM, Eraky I, El-Far MA, Osman HG, Ghoneim MA (2000) Rapid diagnosis of genitourinary tuberculosis by polymerase chain reaction and non-radioactive DNA hybridization. J Urol 164: 584-588.

8. Arisan S, Sonmez NC, Cakir OO, Ergenekon E (2003) Polymerase chain reaction is a good diagnostic tool for Mycobacterium tuberculosis in urine samples. J Cell Mol Biol 2: 99-103.

9. Khatonabadi M, Kim HJ, Lu P, McMillan KL, Cagnon $\mathrm{CH}$, et al. (2013) The feasibility of a regional CTDIvol to estimate organ dose from tube current modulated CT exams. Med Phys 40: 051903.

10. Kapoor R, Ansari MS, Mandhani A, Gulia A (2008) Clinical presentation and diagnostic approach in cases of genitourinary tuberculosis. Indian J Urol 24: 401-405.

11. Abdissa S, Abebe T, Ameni G, Teklu S, Bekuretsion Y, et al. (2018) Endometrial tuberculosis among patients undergoing endometrial biopsy at Tikur Anbesa specialized hospital, Addis Ababa, Ethiopia. BMC Infect Dis 18: 304.

12. Lewinsohn DM, Leonard MK, LoBue PA, Cohn DL, Daley CL, et al. (2017) Official American Thoracic Society/Infectious Diseases Society of America/Centers for Disease Control and Prevention Clinical Practice Guidelines: Diagnosis of Tuberculosis in Adults and Children. Clin Infect Dis 64: 111115.

13. Kim JK, Bang WJ, Oh CY, Yoo C, Cho JS (2013) Feasibility of the interferon- $\gamma$ release assay for the diagnosis of genitourinary tuberculosis in an endemic area. Korean $\mathrm{J}$ Urol 54: 123-126.

14. Sharma JB (2015) Current Diagnosis and Management of Female Genital Tuberculosis. J Obstet Gynaecol India 65: 362-371. 